\title{
LARGE DEFORMATIONS OF A HEAVY CANTILEVER*
}

\author{
BY \\ CHANG-YI WANG \\ Michigan State University
}

\begin{abstract}
A cantilever of uniform cross-section and density is held at an angle $\alpha$ at one end. The shape of the cantilever depends heavily on $\alpha$ and a nondimensional parameter $K$ which represents the relative importance of density and length so that of flexural rigidity. Perturbations on the elastica equations for small and large $K$ show good agreement with exact numerical integration. It is found that whenever $K$ reaches a critical value, bifurcations of the solutions occur. This nonuniqueness can be observed by the flipping phenomena as $\alpha$ is increased.
\end{abstract}

Introduction. A cantilever deformed by its own weight is of interest both practically due to its engineering significance, and theoretically due to its inherent nonlinearity. If the cantilever is thin enough, its deformed shape can be described by the elastica theory. Using this approximation and small deflections, Euler first investigated the stability of a vertical cantilever (column) under its own weight [1]. Euler's stability problem was later corrected by Greenhill [2] who obtained the minimum unstable height for a column of given density and rigidity. The large deformations of a heavy elastica was first numerically integrated by Bickley [3] who, as we shall see later, found only one of the solutions of the originally horizontal cantilever.

This paper is a study in depth of the heavy elastica cantilever. The physical problem is illustrated in Fig. 1, where a cantilever of uniform density and cross-section is anchored on one end. We ask, what is the relation between the end angle $\alpha$ and the end torque $M^{\prime}$ ? Both Greenhill's and Bickley's results will be special cases of this study.

Formulation. Let us consider a small segment of an elastica with total length $L$ and density $\rho$ (Fig. 1). A moment balance gives

$$
m-\rho\left(L-s^{\prime}\right) \sin \theta d s^{\prime}=m+d m
$$

where $m$ is the local moment, $s^{\prime}$ is the arc length from the origin and $\theta$ is the local angle of inclination. According to Euler, the local moment is proportional to the curvature

$$
m=-E I \frac{d \theta}{d s^{\prime}} .
$$

\footnotetext{
* Received June 3, 1980.
} 

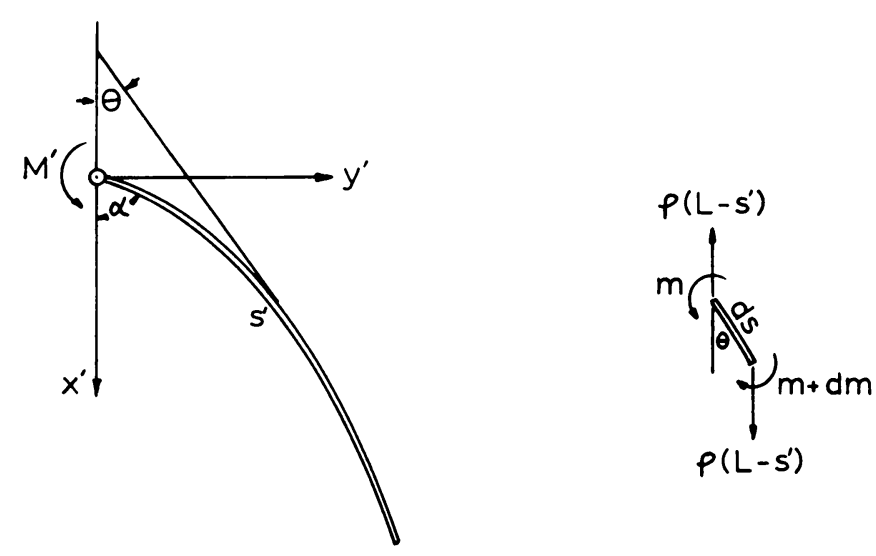

FIG. 1. The coordinate system.

Here $E I$ is the flexural rigidity. Eqs. $(1,2)$ give

$$
E I \frac{d^{2} \theta}{d s^{\prime 2}}=\rho\left(L-s^{\prime}\right) \sin \theta
$$

and the boundary conditions are

$$
s^{\prime}=0, \quad \theta=\alpha ; \quad s^{\prime}=L, \quad d \theta / d s^{\prime}=0
$$

The shape of the elastica is given by

$$
d x^{\prime} / d s^{\prime}=\cos \theta, \quad d y^{\prime} / d s^{\prime}=\sin \theta, \quad x^{\prime}(0)=y^{\prime}(0)=0,
$$

where $x^{\prime}$ and $y^{\prime}$ are cartesian coordinates. If we normalize all lengths by $L$ and drop primes, Eq. (3) becomes

$$
\frac{d^{2} \theta}{d s^{2}}=K^{3}(1-s) \sin \theta
$$

The important parameter $K \equiv\left(\rho L^{3} / E I\right)^{1 / 3}$ represents the relative importance of density and length to that of flexural rigidity.

Series solution for small $K$. Small $K$ means a relatively rigid material and we expect the local angle to differ little from the end angle $\alpha$. We set

$$
\theta=\alpha+K^{3} \theta_{1}+K^{6} \theta_{2}+K^{9} \theta_{3}+\cdots
$$

Eqs. $(4,6)$ yield the successive equations

$$
\begin{aligned}
& d^{2} \theta_{1} / d s^{2}=(1-s) \sin \alpha, \quad \theta_{1}(0)=\frac{d \theta_{1}}{d s}(1)=0 \\
& d^{2} \theta_{2} / d s^{2}=(1-s) \theta_{1} \cos \alpha, \quad \theta_{2}(0)=\frac{d \theta_{2}}{d s}(1)=0, \\
& d^{2} \theta_{3} / d s^{2}=(1-s)\left[\theta_{2} \cos \alpha-\left(\theta_{1}^{2} / 2\right) \sin \alpha\right], \quad \theta_{2}(0)=\frac{d \theta_{2}}{d s}(1)=0 .
\end{aligned}
$$


The solutions are

$$
\begin{aligned}
\theta_{1}= & \frac{\sin \alpha}{6}\left(-s^{3}+3 s^{2}-3 s\right), \\
\theta_{2}= & \frac{\sin \alpha \cos \alpha}{180}\left(s^{6}-6 s^{5}+15 s^{4}-15 s^{3}+9 s\right), \\
\theta_{3}= & \frac{\sin ^{3} \alpha}{72}\left(\frac{s^{9}}{72}-\frac{s^{8}}{8}+\frac{s^{7}}{2}-\frac{11}{10} s^{6}+\frac{27}{20} s^{5}-\frac{3}{4} s^{4}+\frac{9}{40} s\right) \\
& -\frac{\cos ^{2} \alpha \sin \alpha}{60}\left(\frac{s^{9}}{216}-\frac{s^{8}}{24}+\frac{s^{7}}{6}-\frac{s^{6}}{3}+\frac{s^{5}}{4}+\frac{s^{4}}{4}-\frac{s^{3}}{2}+\frac{3}{8} s\right) .
\end{aligned}
$$

The end torque, normalized by $E I / L$, is

$$
\begin{aligned}
M=-\left.\frac{d \theta}{d s}\right|_{s=0}= & \frac{K^{3}}{2} \sin \alpha-\frac{K^{6}}{20} \sin \alpha \cos \alpha \\
& -K^{9}\left(\frac{\sin ^{3} \alpha}{320}-\frac{\cos ^{2} \alpha \sin \alpha}{160}\right)+O\left(K^{12}\right) .
\end{aligned}
$$

The maximum torque is attained when

$$
\alpha=\frac{\pi}{2}+\frac{1}{10} K^{3}-\frac{11}{6000} K^{9}+O\left(K^{12}\right) .
$$

After $\theta(s)$ is found, the shape of the elastica can be easily obtained by integrating

$$
x=\int_{0}^{s} \cos \theta d s, \quad y=\int_{0}^{s} \sin \theta d s
$$

where

$$
\begin{aligned}
\cos \theta= & \cos \alpha-K^{3} \theta_{1} \sin \alpha-K^{6}\left(\frac{\theta_{1}^{2}}{2} \cos \alpha+\theta_{2} \sin \alpha\right) \\
& -K^{9}\left[\theta_{1} \theta_{2} \cos \alpha+\left(\theta_{3}-\frac{\theta_{1}^{3}}{6}\right) \sin \alpha\right]+O\left(K^{12}\right) \\
\sin \theta= & \sin \alpha+K^{3} \theta_{1} \cos \alpha-K^{6}\left(\frac{\theta_{1}^{2}}{2} \sin \alpha-\theta_{2} \cos \alpha\right) \\
& -K^{9}\left[\theta_{1} \theta_{2} \sin \alpha-\left(\theta_{3}-\frac{\theta_{1}^{3}}{6}\right) \cos \alpha\right]+O\left(K^{12}\right)
\end{aligned}
$$

Asymptotic solution for large $K$. The cantilever is relatively compliant if $K$ is large. We set

$$
K^{3} \equiv \delta^{-2} \gg 1
$$

where $\delta$ is small. Eq. (6) then becomes a singular perturbation problem

$$
\delta^{2}\left(d^{2} \theta / d s^{2}\right)=(1-s) \sin \theta
$$


The interior solution is

$$
\sin \theta=0 \text { or } \theta= \pm n \pi \text {. }
$$

For the " boundary layer" near $s=0$ we set

and Eq. (15) becomes

$$
s \equiv \delta t
$$

$$
d^{2} \theta / d t^{2}=(1-\delta t) \sin \theta
$$

The boundary conditions are

$$
t=0, \quad \theta=\alpha ; \quad t \rightarrow \infty, \quad \theta \rightarrow \text { interior solution. }
$$

If we expand $\theta$ in terms of $\delta$,

$$
\theta=\varphi_{0}(t)+\delta \varphi_{1}(t)+\cdots
$$

the zeroth-order equation is

$$
d^{2} \varphi_{0} / d t^{2}=\sin \varphi_{0} .
$$

Multiplying by $d \varphi_{0} / d t$ and integrating results in

$$
\frac{1}{2}\left(\frac{d \varphi_{0}}{d t}\right)^{2}=-\cos \varphi_{0}+C_{1} .
$$

After matching with the interior solution, we find that the constant $C_{1}$ is unity and, for boundary-layer-type solutions to exist, $n$ can only be even. A further integration yields only two continuous solutions:

$$
\begin{aligned}
& n=0, \quad t=-\ln \left(\tan \frac{\varphi_{0}}{4} / \tan \frac{\alpha}{4}\right), \\
& n=2, \quad t=-\ln \left(\tan \frac{\varphi_{0}-2 \pi}{4} / \tan \frac{\alpha-2 \pi}{4}\right) .
\end{aligned}
$$

The first-order equation is

$$
d^{2} \varphi_{1} / d t^{2}=-t \sin \varphi_{0}+\varphi_{1} \cos \varphi_{0}, \quad \varphi_{1}(0)=0, \quad \varphi_{1}(\infty) \rightarrow 0
$$

Eq. (24) is not easy to solve as it stands. However, Eq. (23) suggests the following transform:

$$
\theta \equiv 4 \tan ^{-1} u+n \pi, \quad n=0,2 .
$$

Then Eqs. $(18,19)$ become

$$
\begin{gathered}
\left(1+u^{2}\right) \frac{d^{2} u}{d t^{2}}-2 u\left(\frac{d u}{d t}\right)^{2}=(1-\delta t) u\left(1-u^{2}\right), \\
u(0)=C, \quad u(\infty)=0
\end{gathered}
$$

where

$$
\begin{array}{rlrl}
C & \equiv \tan \frac{\alpha}{4}, & n=0 \\
& \equiv \tan \frac{\alpha-2 \pi}{4}, & & n=2 .
\end{array}
$$


Now we expand

$$
u=u_{0}+\delta u_{1}+\cdots
$$

Eq. (26) yields

$$
\begin{gathered}
\left(1+u_{0}^{2}\right) \frac{d^{2} u_{0}}{d t^{2}}-2 u_{0}\left(\frac{d u_{0}}{d t}\right)^{2}=u_{0}\left(1-u_{0}^{2}\right), \quad u_{0}(0)=C, \quad u_{0}(\infty)=0, \\
\left(1+u_{0}^{2}\right) \frac{d^{2} u_{1}}{d t^{2}}+2 u_{0} u_{1} \frac{d^{2} u_{0}}{d t^{2}}-2 u_{1}\left(\frac{d u_{0}}{d t}\right)^{2}-4 u_{0} \frac{d u_{0}}{d t} \frac{d u_{1}}{d t}=u_{1}-3 u_{0}^{2} u_{1}-t\left(u_{0}-u_{0}^{3}\right) \\
u_{1}(0)=0, \quad u_{1}(\infty)=0 .
\end{gathered}
$$

The solution of Eq. (30) is Eq. (23),

$$
u_{0}=C e^{-t} .
$$

The form of $u_{0}$ suggests that $u_{1}$ should also be exponential. We find

$$
u_{1}=\frac{C}{4}\left(t+t^{2}\right) e^{-t}-\frac{C^{3}}{8}\left(e^{-3 t}-e^{-t}\right)
$$

We shall stop at $u_{1}$, although it is possible to obtain $u_{2}$. The end torque is

$$
\begin{aligned}
M=-\left.\frac{d \theta}{\delta d t}\right|_{t=0} & =-\left.\frac{1}{\delta} \frac{d u}{d t} \frac{d \theta}{d u}\right|_{t=0}=-\frac{4}{\delta\left(1+C^{2}\right)}\left[-C+\frac{\delta}{4}\left(C+C^{3}\right)+O\left(\delta^{2}\right)\right] \\
& = \begin{cases}\frac{1}{\delta}\left[2 \sin \frac{\alpha}{2}-\delta \tan \frac{\alpha}{4}+O\left(\delta^{2}\right)\right] & n=0 \\
\frac{1}{\delta}\left[2 \sin \frac{\alpha-2 \pi}{2}-\delta \tan \frac{\alpha-2 \pi}{2}+O\left(\delta^{2}\right)\right], & n=2 .\end{cases}
\end{aligned}
$$

The maximum torque is at

$$
\alpha=\pi-(-1)^{n / 2} \delta+O\left(\delta^{2}\right) .
$$

Stability of a slightly inclined column. Suppose $K$ is arbitrary. Eq. (6) admits two exact solutions, $\theta=\alpha=0$ and $\theta=\alpha=\pi$. Perturbations about the latter solution yields the stability properties of a column. We set

$$
\alpha=\pi+\varepsilon, \quad \theta=\pi+\varepsilon \psi_{1}(s)+\varepsilon^{2} \psi_{2}(s)+\varepsilon^{3} \psi_{3}(s)+\cdots .
$$

Eqs. $(4,6)$ yield

$$
d^{2} \psi_{1} / d s^{2}=-K^{3}(1-s) \psi_{1}, \quad \psi_{1}(0)=1, \quad\left(d \psi_{1} / d s\right)(1)=0 .
$$

Let

$$
z=K(1-s) .
$$

Then Eq. (37) reduces to

$$
\left(d^{2} \psi_{1} / d z^{2}\right)+z \psi_{1}=0
$$


This is known as the Stokes equation. The solution is

$$
\psi_{1}=c_{1} z^{1 / 2} J_{-1 / 3}\left(\frac{2}{3} z^{3 / 2}\right)+c_{2} z^{1 / 2} J_{1 / 3}\left(\frac{2}{3} z^{3 / 2}\right) .
$$

Using the boundary conditions

we find

$$
z=0, \quad d \psi_{1} / d z=0 ; \quad z=K, \quad \psi_{1}=1,
$$

$$
\psi_{1}=\frac{z^{1 / 2} J_{-1 / 3}\left(\frac{2}{3} z^{3 / 2}\right)}{K^{1 / 2} J_{-1 / 3}\left(\frac{2}{3} K^{3 / 2}\right)} .
$$

Note that Eq. (42) is unbounded when $\frac{2}{3} K^{3 / 2}$ is a zero of $J_{-1 / 3}$. The zeros are at 1.86635 , $4.98830,8.12437,11.26355$, etc. The corresponding values of $K$ are 1.98635, 3.82557, $5.29566,6.58432$, etc. These are the critical values near which a small change $\varepsilon$ would cause large changes in shape. The first zero was found by Greenhill [2] using linear theory. He concluded that if $K<1.986$ the column is stable.

The equations for the higher-order corrections are

$$
\begin{aligned}
& d^{2} \psi_{2} / d s^{2}=-K^{3}(1-s) \psi_{2}, \quad \psi_{2}(0)=0, \quad\left(d \psi_{2} / d s\right)(1)=0, \\
& d^{2} \psi_{3} / d s^{2}=-K^{3}(1-s)\left(\psi_{3}-\frac{\psi_{1}^{3}}{6}\right), \quad \psi_{3}(0)=0, \quad\left(d \psi_{3} / d s\right)(1)=0 .
\end{aligned}
$$

We can safely say $\psi_{2} \equiv 0$. However, it is probably not worth while to seek the solution for $\psi_{3}$. The end torque is

$$
M=-\left.\frac{d \theta}{d s}\right|_{s=0}=\left.\varepsilon K \frac{d \psi_{1}}{d z}\right|_{z=K}+O\left(\varepsilon^{3}\right)=-\varepsilon K^{3 / 2} \frac{J_{2 / 3}\left(\frac{2}{3} K^{3 / 2}\right)}{J_{-1 / 3}\left(\frac{2}{3} K^{3 / 2}\right)}+O\left(\varepsilon^{3}\right) .
$$

Exact numerical integration. Bickley [3], using a rather tedious numerical method, integrated the cases for $\alpha=\pi / 2, K<2.44$. He obtained only one solution. Lippmann, Mahrenholtz and Johnson [4] reproduced Bickley's results with an analogue computer. We shall introduce here a simple method to investigate the nonuniqueness associated with large $K$.

Let

$$
r \equiv K(1-s)
$$

and Eq. (6) becomes

$$
d^{2} \theta / d r^{2}=r \sin \theta .
$$

We choose any value for $\theta$ at $r=0$. Using the boundary condition

$$
\left.\frac{d \theta}{d r}\right|_{r=0}=0
$$

Eq. (47) is integrated as an initial value problem by the fourth-order Runge-Kutta algorithm. Every point along the integration path is a solution. Suppose we stop at $r=r^{*}$, say; then

$$
\begin{aligned}
& K=r^{*}, \quad \alpha=\theta\left(r^{*}\right), \\
& M=-\left.\frac{d \theta}{d s}\right|_{s=0}=r^{*} \frac{d \theta}{d r}\left(r^{*}\right) .
\end{aligned}
$$


Thus we obtain the end torque $M$ as a function of $\alpha$. If the shape of the cantilever is needed, we can integrate Eq. (6) and

$$
d x / d s=\cos \theta, \quad d y / d s=\sin \theta
$$

with the initial conditions Eq. (50) and

$$
x(0)=y(0)=0, \quad \theta(0)=\alpha .
$$

Results and discussion. Using our numerical scheme we integrated the heavy elastica cantilever problem for $K \leq 6$ and arbitrary $\alpha$. Partial results are shown in Fig. 2 where the end torque $M$ is plotted as a function of $\alpha$. Only half of the curves are shown, since they are

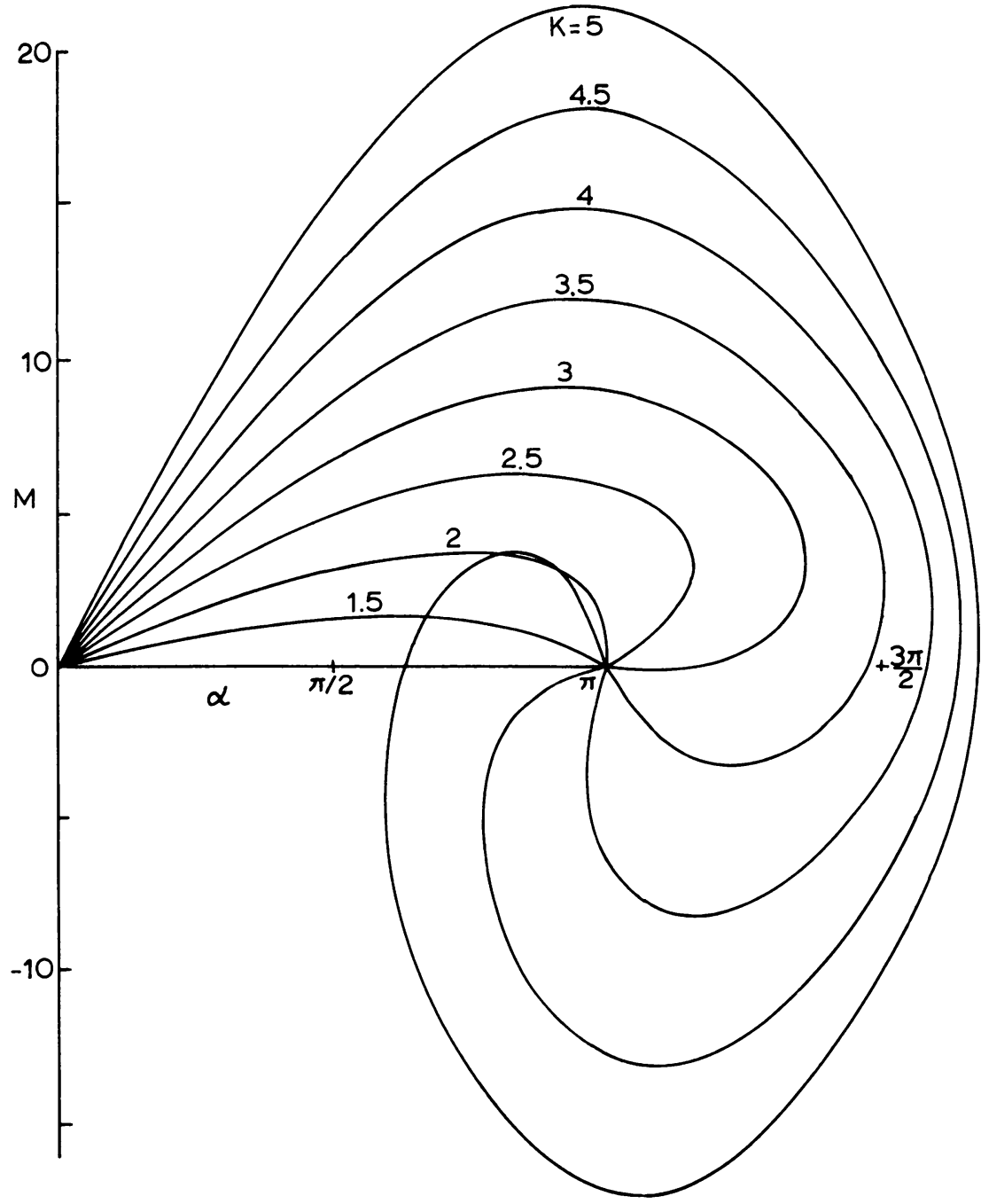

Fig. 2. End torque as a function of end angle. 


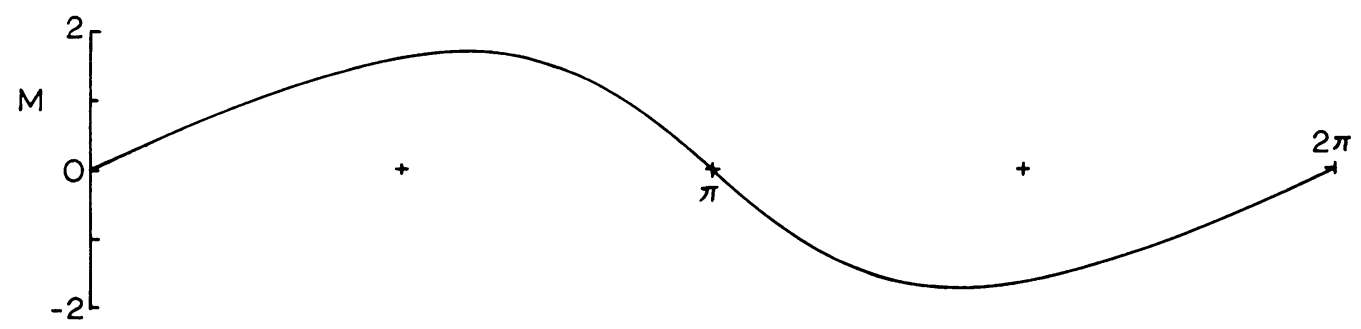

FIG. 3. The complete torque curve for $K=1.5$.

radially symmetric to the point $M=0, \alpha=\pi$. Note that, as $K$ is increased, the curves change nonlinearly from $\sin \alpha$ (Eq. (10)) to $\sin (\alpha / 2)$ (Eq. (34)).

The complete torque curve for $K=1.5$ is shown in Fig. 3. The small- $K$ approximation, Eq. (10), is indistinguishable from the exact solution. The solution is unique for $K \leq 1.98635$.

Three solutions occur near $\alpha=\pi$ for $1.98635<K \leq 3.82557$. A typical torque curve $(K=2.5)$ is shown in Fig. 4. As $\alpha$ is increased from zero the equilibrium states follow $A B C D$. Then there is a sudden jump from $D$ to $F$ where the cantilever is flipped to the other side (torque changes sign). In this flipping process energy is dissipated through oscillations. As $\alpha$ is further increased the equilibrium states follow FGA smoothly. The shapes of the cantilever at various states are shown in Fig. 5. If $\alpha$ is decreased, the equilibrium states follow $A G F E C B A$, enclosing a hysteresis loop $D F E C$.

When $3.82557<K \leq 5.29566$, five solutions appear in the neighborhood of $\alpha=\pi$. Fig. 6 shows the complete torque curve for $K=5$. We see that our asymptotic expansion for

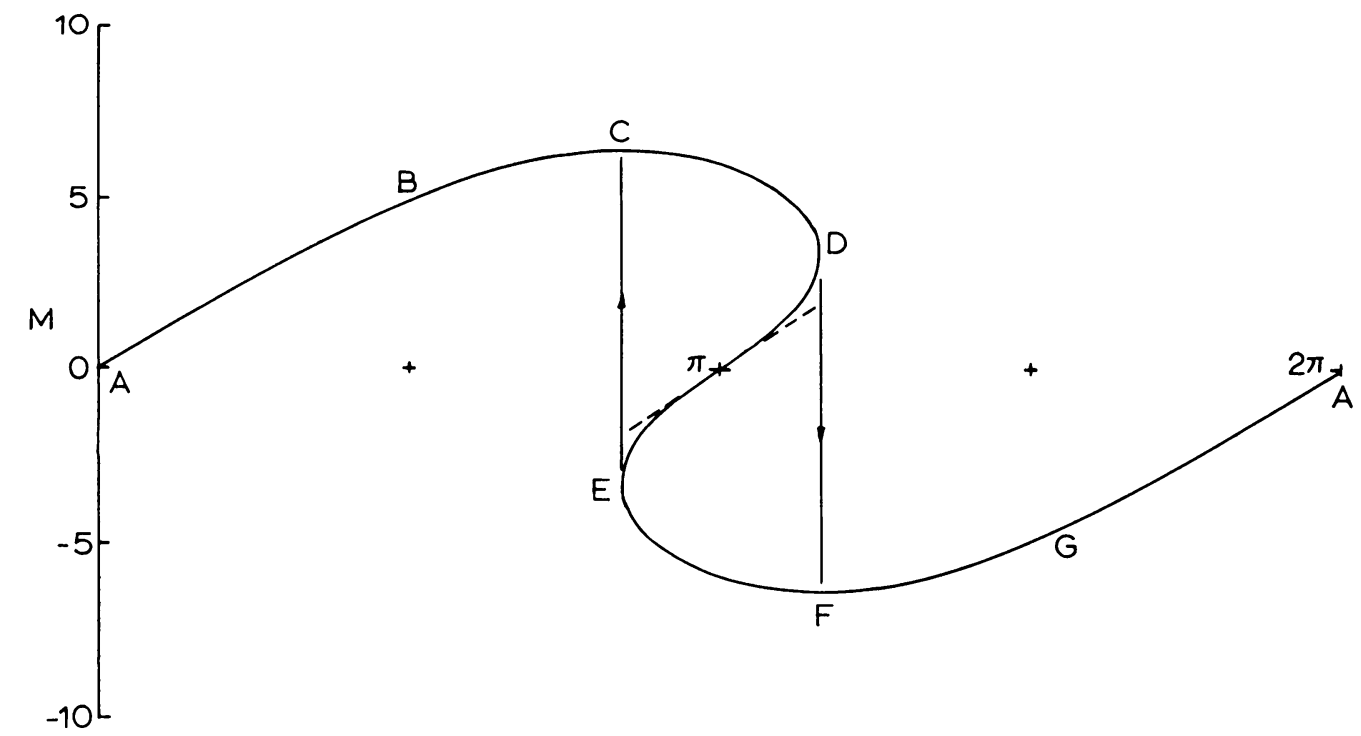

FIG. 4. The complete torque curve for $K=2.5$. Dashed line is Eq. (45). 


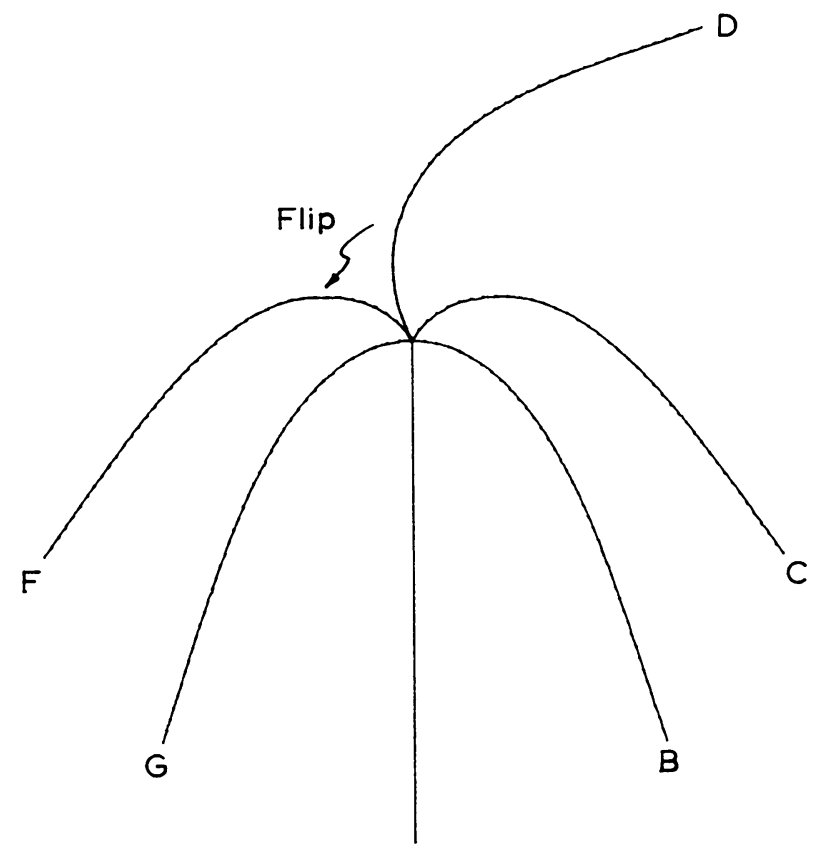

A

Fig. 5. Cantilever shapes for $K=2.5$. Letters correspond to those in Fig. 4 .

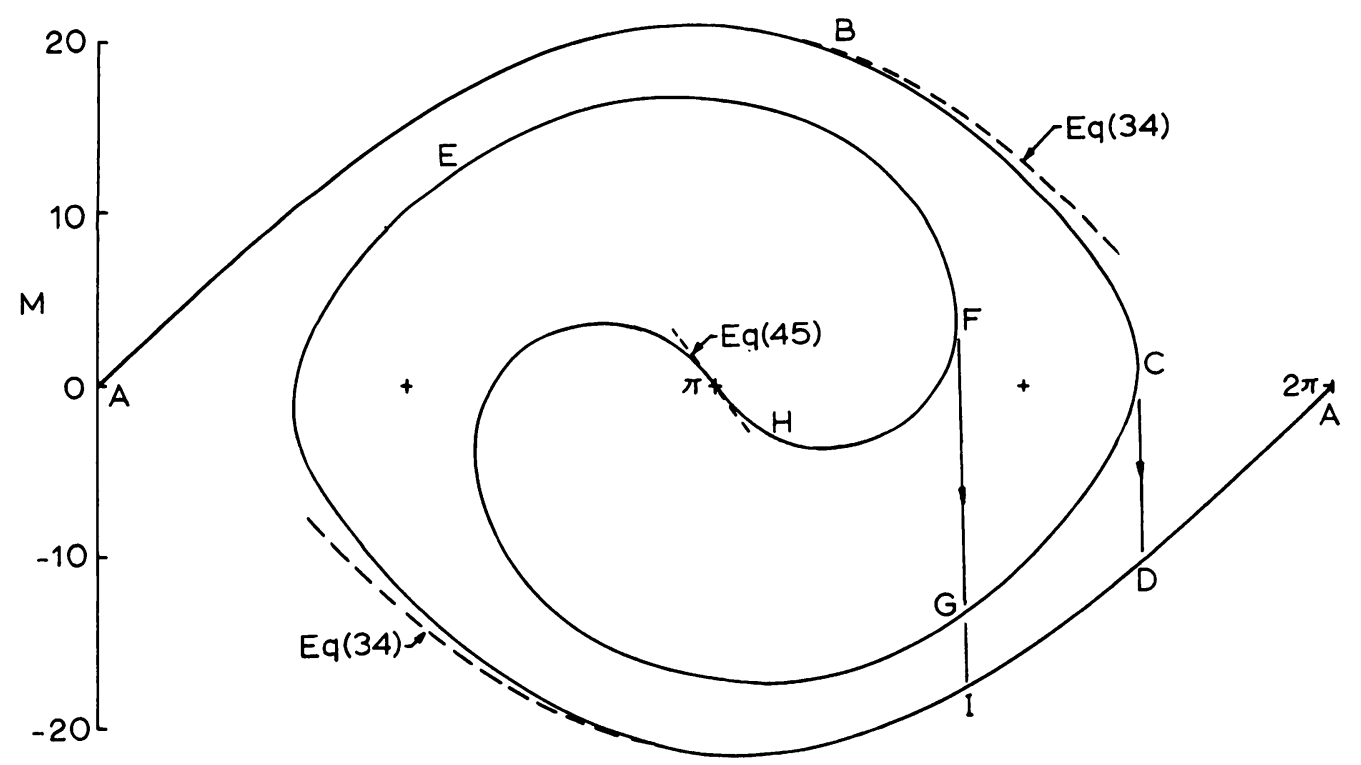

Fig. 6. The complete torque curve for $K=5$. Dashed lines are approximate solutions. 


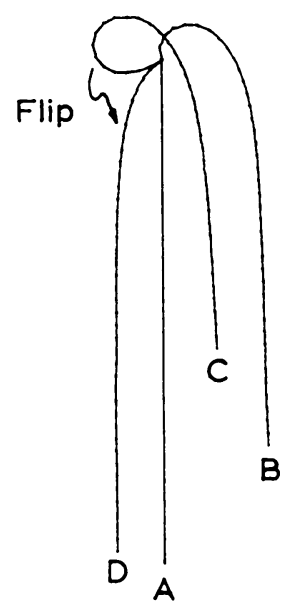

Fig. 7. Cantilever shapes for the primary states, $K=5$. Letters correspond to those in Fig. 6 .

large $K$, Eq. (34), predicts the outermost curves which represent the most stable states. The expansion Eq. (45) approximates the least stable states near $\alpha=\pi, M=0$. As $\alpha$ is increased from zero we expect the equilibrium states to follow $A B C D A$ with a "flip" from $C$ to $D$. This is illustrated by the cantilever configurations in Fig. 7. The boundary layer character is evident for these states which we call the primary states. It is conjectured that the inner curves or the secondary states are locally stable; that is, if exterior perturbations are small enough, the torque curve will be followed. If we start from the state $E$ and increase $\alpha$, a secondary flip occurs from $F$ to $G$ or from $F$ to $I$. Some secondary configurations are shown in Fig. 8. Fig. 9 shows the angle of flip, which is also the boundary of the hysteresis loop. We

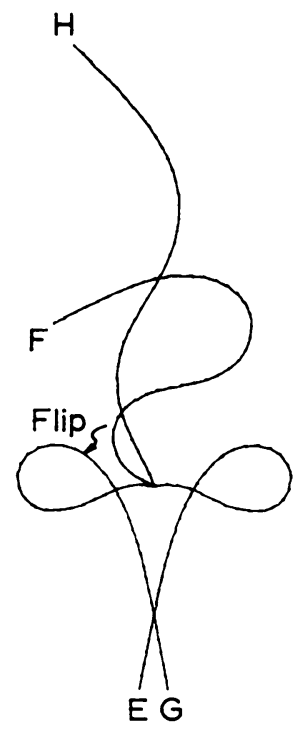

FiG. 8. Cantilever shapes for the secondary states, $K=5$. Letters correspond to those in Fig. 6 . 


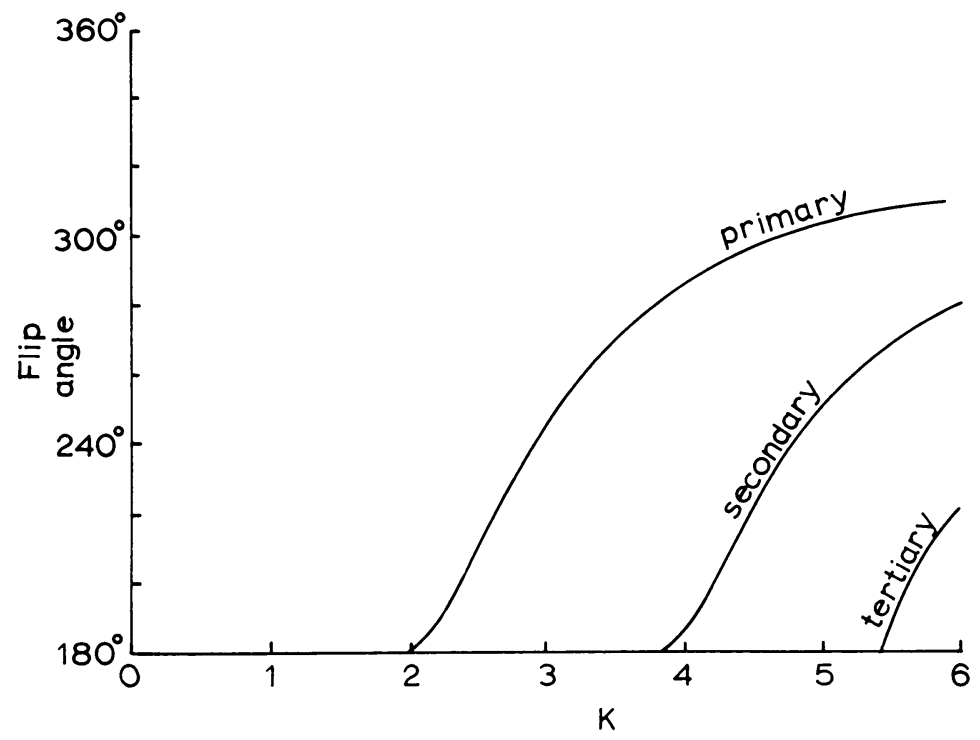

FIG. 9. The angle of flip as a function of $K$.

shall call these the primary, secondary and tertiary flipping angles corresponding to the first, second, and third critical values of $K$.

Fig. 10 shows the maximum torque and the angle at which it occurs as a function of $K$. We see that, within 5\% error, our small- $K$ expansion (Eqs. $(10,11)$ ) is valid for $K \leq 1.5$. The fact that the constant coefficients in Eq. (10) are progressively smaller enables us to extend slightly the radius of convergence of $K$. The asymptotic expansion, Eqs. $(34,35)$, is valid for

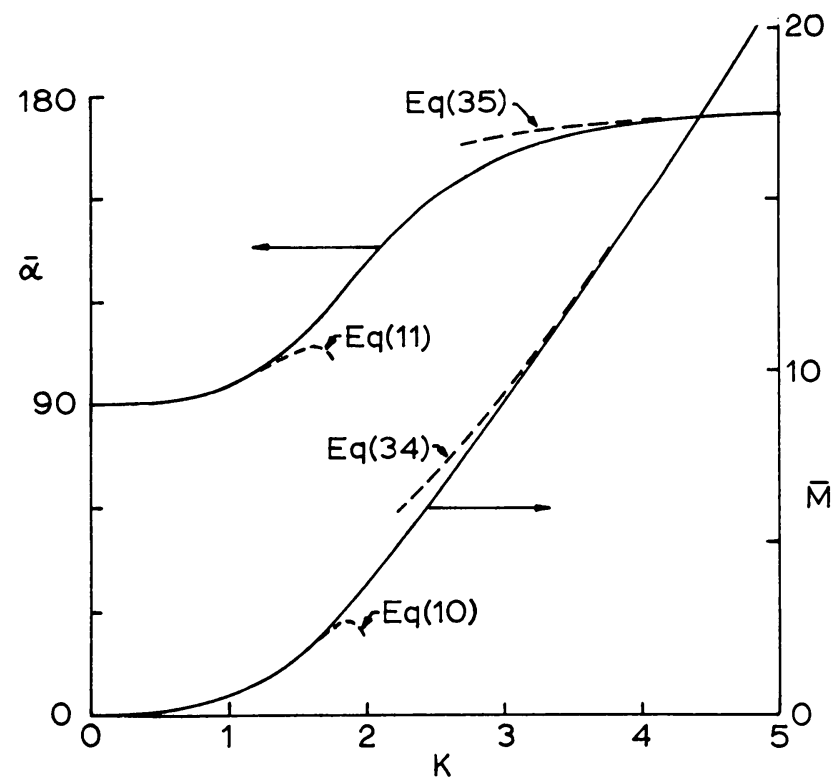

Fig. 10. The maximum torque $\bar{M}$ and the angle at which it occurs $\bar{\alpha}$. 


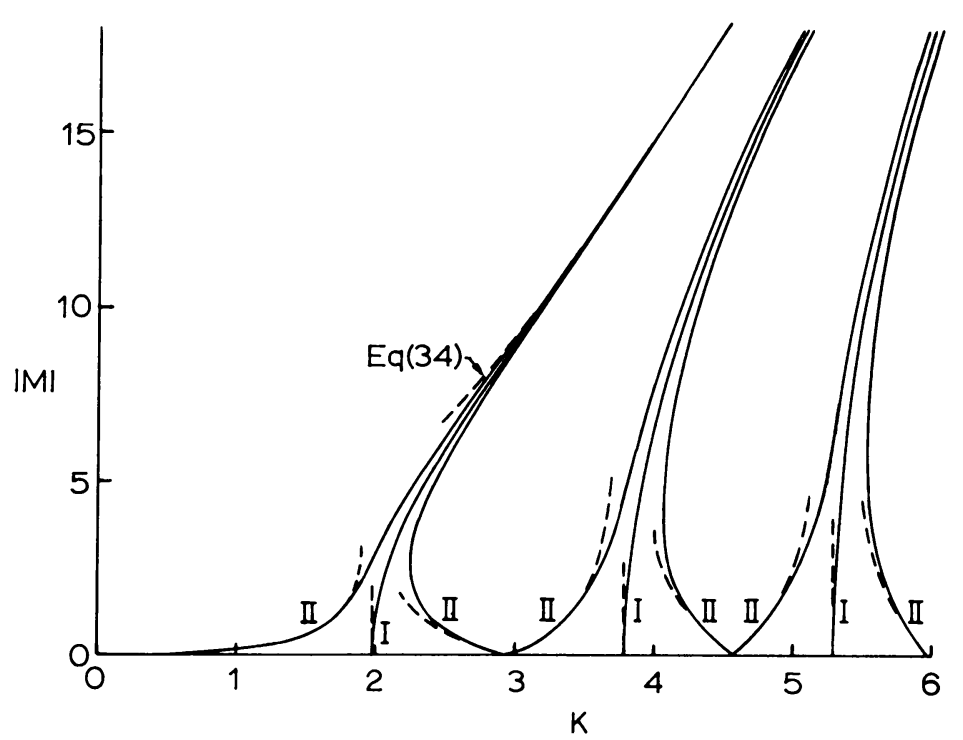

FIG. 11. End torque as a function of $K$ for the vertical and the slightly inclined column. I: $\alpha=180^{\circ}, \mathrm{II}: \alpha=180^{\circ}$ $\pm 10^{\circ}$. Dashed lines are from Eq. (45) unless otherwise noted.

$K$ greater than about 3. In Fig. 11 the torque is plotted as a function of $K$ for $\alpha=180^{\circ}$ (the vertical column and $\alpha=180^{\circ} \pm 10^{\circ}$ (the slightly inclined column). The validity of Eq. (45) is limited to $|M|<1$, while Eq. (34) predicts only the primary state. The exact results also show the torque is never infinite at the critical values of $K$. If we define nonlinear instability as where nonuniqueness may occur, or where small changes in $K$ invoke large changes in $M$, then for $\alpha=180^{\circ}$ the stability criterion is $K<1.98635$ and for $\alpha=180^{\circ} \pm 10^{\circ}$, the stability criterion is $K<2.5$. This dependence is the same as the primary flipping curve shown in Fig. 10. The inclined column is more stable than the vertical column!

Lastly, we comment on the approximate method whereby $\theta$ in Eq. (47) is expanded as a power series in $r$ and boundary conditions are applied at both $r=0$ and $r=1[5,6,7]$. There is no mathematical justification that this series must converge at $r=1$ and indiscriminate application of this method may lead to large errors, since the important parameters $\alpha$ and $M$ are evaluated at $r=1$. Our study shows that the only time when a power series is a valid approximation is when $K$ is small. Thus if we expand $\theta$ in a Taylor series up to $r^{9}$ as in our Eq. (7), the solution is theoretically sound if $K \ll 1$ and our exact results show $K$ may be extended to 1.5 at most. The proper way, of course, is to expand in terms of $K$ as in Eq. (7).

\section{REFERENCES}

[1] L. Euler, De curvis elasticis (1744)

[2] A. G. Greenhill, Determination of the greatest height consistent with stability that a vertical pole or mast can be made, and of the greatest height to which a tree of given proportions can grow, Proc. Camb. Phil. Soc. 4, 65-73 (1881)

[3] W. G. Bickley, The heavy elastica, Phil. Mag. Ser. 7 17, 603-622 (1934)

[4] H. Lippmann, O. Mahrenholtz and W. Johnson, Thin heavy elastic strips at large deflexions, Int. J. Mech. Sci. 2, 294-310(1961) 
[5] F. V. Rhode, Large deflections of a cantilever beam with uniformly distributed load, Quart. Appl. Math. 11, 337-338 (1953)

[6] R. Frisch-Fay, The analysis of a vertical and a horizontal cantilever under a uniformly distributed load, J. Franklin Inst. 271, 192-199(1961)

[7] R. Schmidt and D. A. DaDeppo, Approximate analysis of large deflections of beams, Zeit. angew. Math. Mech. 51, 233-234 (1971) 